\title{
Study of the development of plastic instabilities during tests on metallic plates biaxially loaded in their plane, in tension or in compression
}

\author{
DOMINIque Jouve ${ }^{\text {a }}$ \\ CEA, DAM, DIF, 91297 Arpajon, France
}

Received 15 June 2015, Accepted 18 November 2015

\begin{abstract}
Plastic instabilities develop during tension and compression tests on metallic plates biaxially loaded in their plane. They limit the acceptable deformation levels during sheet forming. Carrying out a Linear Stability Analysis, we study the onset of their development. We calculate the growth rate of small symmetrical and antisymmetrical defects with respect to the median plane of the plate, periodic along the loading directions, and we determine the dominant mode. This $3 D$ model applies to dynamic tests whatever the thickness. It retrieves classical results for thin plates statically loaded in tension. Plane tension and compression tests are two particular $2 D$ cases of this model. In plane strain tension on ductile non viscous materials, we retrieve that the first instabilities, which are also long wavelength necking ones, arise very little time before the applied force is maximum; this is consistent with the experimental observations of Considère. As time goes by, antisymmetric modes with shorter wavelengths compete with the symmetric ones.
\end{abstract}

Key words: Plastic instabilities / linear stability analysis / tension and compression tests / biaxial loading / symmetric and antisymmetric defects

\section{Introduction}

Plastic instabilities develop during tension and compression tests on metallic plates biaxially loaded in their plane. They limit the acceptable deformation levels during sheet forming. Many publications have dealt with the onset and the development of necking in tension, since the pioneering works of Considère (1885) [1]. Many of them deal with thin plates statically loaded [2-9].

In order to study the onset of the development of these instabilities, we carry out a Linear Stability Analysis $[7,8,10-15]$. We consider a plate dynamically loaded with constant velocities applied at its edges (our model applies whatever the thickness) (see Fig. 1), and we calculate the growth-rate $\theta$ of small perturbations $\delta \vec{x}$ of the material trajectories of the mean homogeneous flow of the perfect plate (cf. [15], Chap. 7) ${ }^{1}$, that are representative of the instabilities. We suppose that they grow exponentially

\footnotetext{
a Corresponding author: dominique.jouve@cea.fr

1 For an incompressible material, the projection on the $x_{1}$ and $x_{2}$-loading directions of the velocity of each material particle remains constant with time. The velocity gradients along these directions are uniform at each time (but they evolve with time).
}

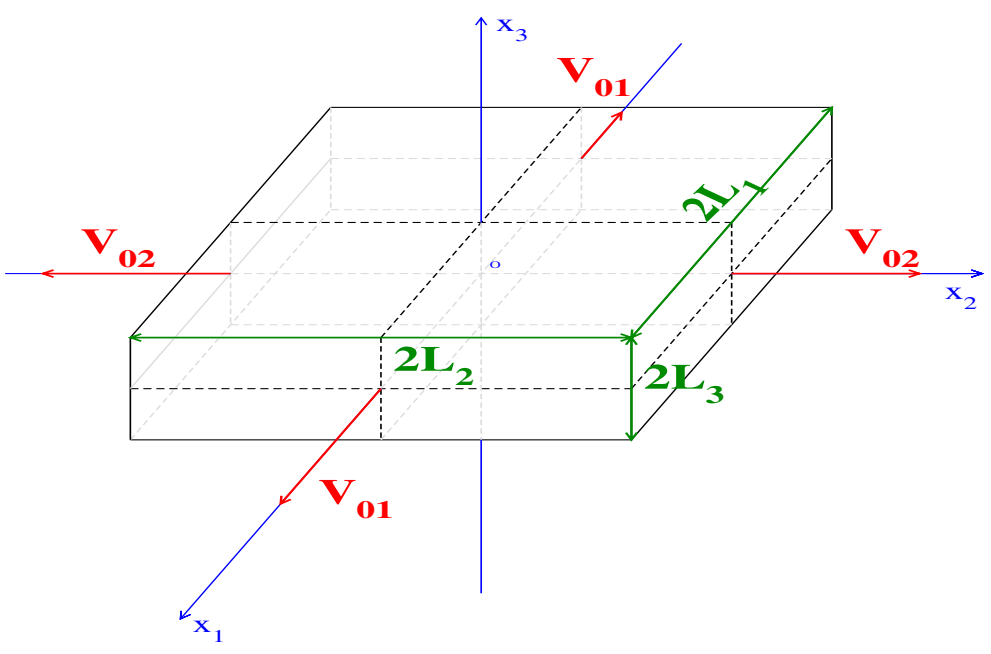

Fig. 1. Plate biaxially loaded in tension in its plane, with constant velocities applied at its edges.

$\left(\delta \vec{x}=e^{\theta t} \times \vec{F}(\right.$ space variables $\left.)\right)$. They are supposed to be periodic along the $x_{1}$ - and $x_{2}$-loading directions (we denote respectively by $\lambda_{1}$ and $\lambda_{2}$ the wavelength along the $x_{1}$ - and $x_{2}$-axes, and by $\gamma_{i}=2 \pi / \lambda_{i}(i=1,2)$ the 


\section{Nomenclature}

\begin{tabular}{|c|c|c|}
\hline \multicolumn{3}{|c|}{ Latin symbols } \\
\hline$C_{v}$ & Isochoric heat capacity & $\mathrm{J} . \mathrm{kg}^{-1} \cdot \mathrm{K}^{-1}$ \\
\hline$D_{i j}$ & Component of strain-rate tensor $\mathbf{D}$ & $\mathrm{s}^{-1}$ \\
\hline$D_{i j}^{p}$ & Component of plastic strain-rate tensor $\mathbf{D}^{\mathbf{p}}$ & $\mathrm{s}^{-1}$ \\
\hline G & Shear modulus & $\mathrm{Pa}$ \\
\hline$L_{i}$ & Half-dimension of the plate & $\mathrm{m}$ \\
\hline$L_{0 i}$ & Initial half-dimension of the plate & $\mathrm{m}$ \\
\hline$t$ & Time & $\mathrm{s}$ \\
\hline$T$ & Absolute temperature & $\mathrm{K}$ \\
\hline$V_{0 \alpha}$ & Applied velocity & $\mathrm{m} \cdot \mathrm{s}^{-1}$ \\
\hline$x_{0 i}$ & Lagrangian coordinate & $\mathrm{m}$ \\
\hline$S_{i j}$ & Component of the deviatoric part $\mathbf{S}$ of Cauchy stress tensor $\boldsymbol{\Sigma}$ & $\mathrm{Pa}$ \\
\hline$Y$ & Yield strength & $\mathrm{Pa}$ \\
\hline \multicolumn{3}{|c|}{ Greek symbols } \\
\hline$\alpha=D_{22} / D_{11}$ & Velocity gradient ratio & \\
\hline$\gamma_{\alpha}=2 \pi / \lambda_{\alpha}$ & Perturbation wavenumber along $x_{\alpha}$-loading direction & $\mathrm{m}^{-1}$ \\
\hline$\gamma=\sqrt{\gamma_{1}^{2}+\gamma_{2}^{2}}$ & Norm of wave vector $\vec{\gamma}=\gamma_{1} \overrightarrow{e_{1}}+\gamma_{2} \overrightarrow{e_{2}}$ & $\mathrm{~m}^{-1}$ \\
\hline$\delta x_{i}$ & Component of the material trajectories perturbation vector $\delta \vec{x}$ & $\mathrm{~m}$ \\
\hline$\varepsilon_{p}$ & Equivalent plastic strain & \\
\hline$\dot{\varepsilon}_{p}$ & Equivalent plastic strain rate & $\mathrm{s}^{-1}$ \\
\hline$\theta$ & Growth-rate & $\mathrm{s}^{-1}$ \\
\hline$\lambda_{\alpha}$ & Perturbation wavelength along $x_{\alpha}$-loading direction & $\mathrm{m}$ \\
\hline$\rho$ & Mass density & $\mathrm{kg} \cdot \mathrm{m}^{-3}$ \\
\hline$\Sigma_{i j}$ & Component of Cauchy stress tensor $\boldsymbol{\Sigma}$ & $\mathrm{Pa}$ \\
\hline$\psi=\arctan \left(\gamma_{2} / \gamma_{1}\right)$ & Angle between $x_{1}$-axis and wavevector $\vec{\gamma}$ & degree \\
\hline
\end{tabular}

1. subscript $i=1,2,3$

2. subscript $\alpha=1,2$

3. In the manuscript, superscript $(d)$ refers to the dominant mode. For instance, $\lambda_{1}^{(d)}$ denotes the wavelength associated with the dominant mode in $x_{1}$-direction.

4. $\dot{A}$ denotes Lagrangian derivative of $A$, for any quantity $A$.

corresponding wavenumbers). They are symmetric or antisymmetric with respect to the median plane of the plate (cf. Fig. 2). We draw maps $\theta\left(\gamma_{1}, \gamma_{2}\right)$, and we deduce the dominant mode, i.e. the most unstable pair of wavelengths $\left(\lambda_{1}^{(d)}, \lambda_{2}^{(d)}\right)$, and thus we identify the sites where plastic deformation begins to localize.

The material is a metal supposed to be homogeneous, isotropic, incompressible, elastoviscoplastic. Its yield strength $Y$ depends on plastic strain $\varepsilon_{p}$, absolute temperature $T$ and plastic strain-rate $\dot{\varepsilon}_{p}$. Its shear modulus $G$ depends on $T$. The material is supposed to satisfy the Von Mises plasticity criterion $\left(\mathbf{S}: \mathbf{S}=2 Y^{2} / 3\right.$, $\mathbf{S}$ denoting the deviatoric part of Cauchy stress tensor $\boldsymbol{\Sigma}$ ), and the normality flow rule (plastic strain rate tensor $\left.\mathbf{D}^{\mathbf{p}}=\frac{3 \dot{\varepsilon}_{p}}{2 Y} \mathbf{S}\right)$. Its evolution is supposed to be adiabatic, and plastic work to be fully converted into heat. Damage, thermal expansion and heat conduction are neglected.

Linear stability analysis solves the evolution equations of first order perturbations of the mean homogeneous ground flow of the perfect plate: volume and energy conservation equations, lagrangian momentum equations, flow rule, and we write that plastic strain increases with time, so we have simultaneously: $\mathbf{S}: \mathbf{S}=2 Y^{2} / 3$ and $\mathbf{S}: \dot{\mathbf{S}}=2 Y \dot{Y} / 3$.
We have developed a 3D Linear Stability Analysis: it applies to dynamic tests, whatever the thickness of the plate. In tension, when inertial effects are negligible, and for sufficient viscous effects, the wavelength of the most unstable defects is large compared to thickness, and we retrieve the growth-rate of necking plastic instabilities calculated by Dudzinski and Molinari in their Compte Rendu à l'Académie des Sciences in 1988 [7, 8, 16, 17], in the framework of generalized plane stress theory $[18,19]$.

In this paper, first in Section 2 we search for the dominant mode for different loadings; then we deal with plane tension and compression tests in Section 3 (the dimension of the plate is infinite along one loading direction).

\section{Main results of our 3D model}

\subsection{Searching for plastic strain localization lines}

We suppose that the perturbation $\delta \vec{x}$ of material trajectories is periodic along the $x_{1}$ - and $x_{2}$-loading directions, and that the end faces normal to the loading directions remain plane over time. Thus we have (cf. [15], 


\section{Symmetric defects}

cross-section in a plane $\mathrm{x}_{1}=$ constant cross-section in a plane $\mathrm{x}_{2}=$ constant
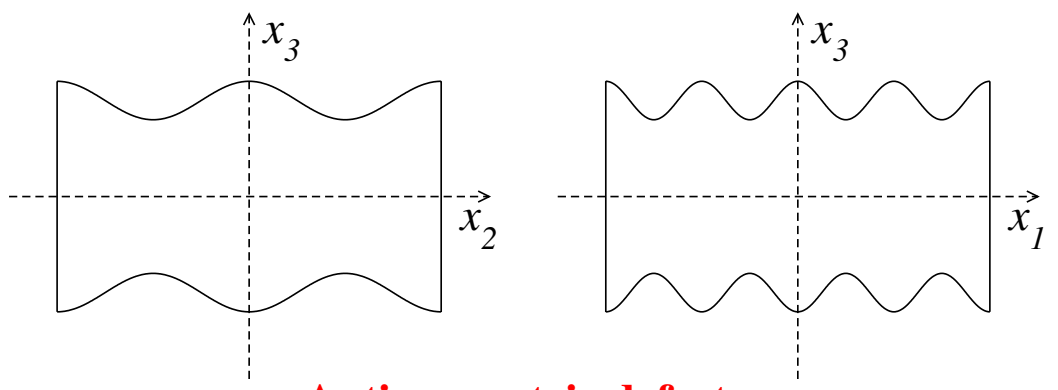

Antisymmetric defects

cross-section in a plane $\mathrm{x}_{1}=$ constant cross-section in a plane $\mathrm{x}_{2}=$ constant
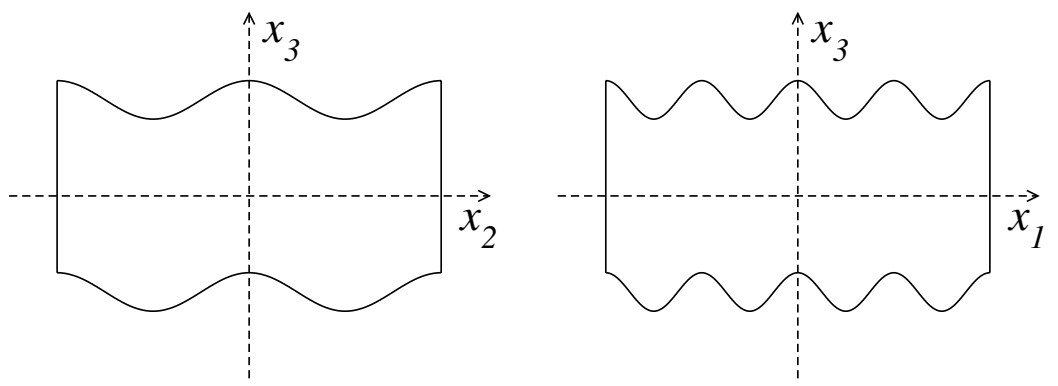

Fig. 2. Symmetric and antisymmetric defects with respect to the median plane of the plate, periodic along the $x_{1}$ - and $x_{2}$-loading directions.

Chap. 18), in Lagrangian coordinates $x_{0 i}(i=1,2,3)$ :

$$
\left\{\begin{array}{l}
\delta x_{1}=\mathrm{e}^{\theta t} \sin \left(\gamma_{1} x_{01}\right) \cos \left(\gamma_{2} x_{02}\right) F_{1}\left(x_{03}\right) \\
\delta x_{2}=\mathrm{e}^{\theta t} \cos \left(\gamma_{1} x_{01}\right) \sin \left(\gamma_{2} x_{02}\right) F_{2}\left(x_{03}\right) \\
\delta x_{3}=\mathrm{e}^{\theta t} \cos \left(\gamma_{1} x_{01}\right) \cos \left(\gamma_{2} x_{02}\right) F_{3}\left(x_{03}\right)
\end{array}\right.
$$

with, setting $L_{0 i}=L_{i}\left(t_{0}=0\right)(i=1,2,3)$ :

$$
\left\{\begin{array}{l}
\gamma_{1} L_{01}=i_{1} \pi\left(i_{1} \in \mathbf{N}\right) \\
\gamma_{2} L_{02}=i_{2} \pi\left(i_{2} \in \mathbf{N}\right)
\end{array}\right.
$$

In the plastic strain localization zones, perturbation $\delta x_{3}$ is extremum (see Fig. 2) (we set: $\delta x_{3, i}=\frac{\partial \delta x_{3}}{\partial x_{0 i}}(i=1,2)$ ):

$$
\left\{\begin{array}{l}
\delta x_{3,1}\left(x_{01}, x_{02}, \pm L_{03}\right)=0 \\
\delta x_{3,2}\left(x_{01}, x_{02}, \pm L_{03}\right)=0
\end{array}\right.
$$

In view of the form given to $\delta \vec{x}$, in the planes at $x_{03}=$ $\pm L_{03}$, plastic strain concentrates along straight lines, defined by the following equations:

$$
\left\{\begin{array}{l}
\gamma_{1} x_{01}+\gamma_{2} x_{02}=K_{+} \pi\left(K_{+} \in \mathbf{N}\right) \\
\gamma_{1} x_{01}-\gamma_{2} x_{02}=K_{-} \pi\left(K_{-} \in \mathbf{N}\right)
\end{array}\right.
$$

The wave vector $\vec{\gamma}=\gamma_{1} \overrightarrow{e_{1}}+\gamma_{2} \overrightarrow{e_{2}}$ is normal to the localization lines "+". The angle between the $x_{2}$-axis and

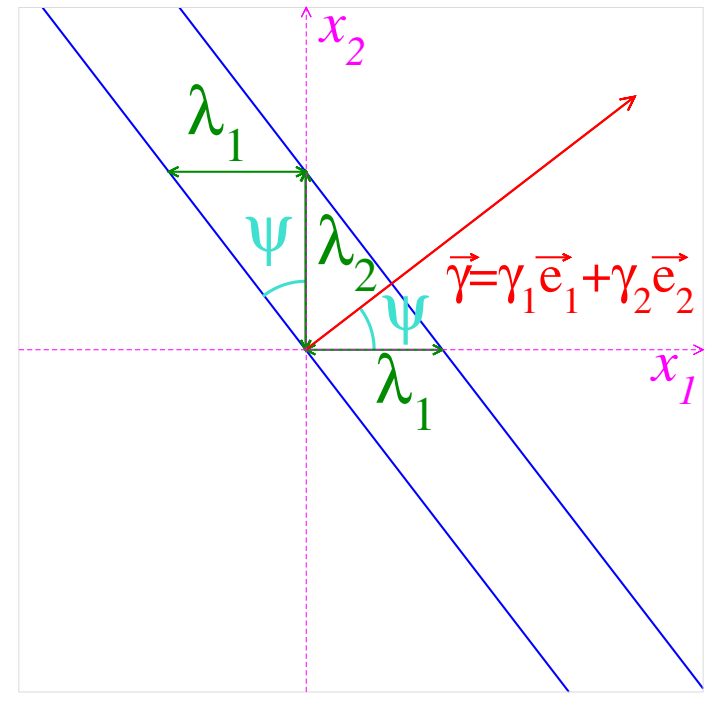

Fig. 3. Two successive localization lines "+": the distance between them equals $\lambda_{1}$ along the $x_{1}$-axis, and $\lambda_{2}$ along the $x_{2}$-axis.

these lines is equal to (see Fig. 3):

$$
\psi=\arctan \left(\frac{\lambda_{1}}{\lambda_{2}}\right)=\arctan \left(\frac{\gamma_{2}}{\gamma_{1}}\right)
$$

(for localization lines "-", this angle equals $-\psi$ ). 
Table 1. Properties of the material.

\begin{tabular}{ccccccccccc}
\hline$\rho$ & $A$ & $B$ & $\varepsilon_{i}$ & $n$ & $C$ & $\dot{\varepsilon}_{0}$ & $m$ & $T_{\text {melt }}$ & $G_{0}$ & $C_{v}$ \\
\hline $5 \mathrm{~g} . \mathrm{cm}^{-3}$ & $1 \mathrm{GPa}$ & $1 \mathrm{GPa}$ & 1 & 0.5 & 0.01 & $1 \mathrm{~s}^{-1}$ & 1.2 & $3000 \mathrm{~K}$ & $100 \mathrm{GPa}$ & $500{\mathrm{~J} . \mathrm{kg}^{-1} . \mathrm{K}^{-1}}$ \\
\hline
\end{tabular}

We illustrate the search for the dominant mode and the plastic strain localization zones for different loadings and for a given material.

\subsection{Material}

Given a (fictitious) metal, whose physical properties are:

1. constant mass density: $\rho=5000 \mathrm{~kg} \cdot \mathrm{m}^{-3}$;

2. its yield strength $Y$ obeys a constitutive law in the form proposed by Johnson and Cook [20], and, as in the model of Steinberg-Cochran-Guinan [21], we suppose that the ratio $Y / G$ is independent of temperature. Thus we have:

$$
\begin{aligned}
Y\left(\varepsilon_{p}, T, \dot{\varepsilon}_{p}\right)= & {\left[A+B\left(\varepsilon_{p}+\varepsilon_{i}\right)^{n}\right]\left[1+C \ln \left(\frac{\dot{\varepsilon}_{p}}{\dot{\varepsilon}_{0}}\right)\right] } \\
& \times\left[1-\left(\frac{T-T_{0}}{T_{\text {melt }}-T_{0}}\right)^{m}\right] \\
G(T)= & G_{0}\left[1-\left(\frac{T-T_{0}}{T_{\text {melt }}-T_{0}}\right)^{m}\right]
\end{aligned}
$$

for:

$\dot{\varepsilon}_{p}>\dot{\varepsilon}_{0}=1 \mathrm{~s}^{-1}$ and $T_{0}=300 \mathrm{~K} \leq T \leq T_{\text {melt }}=3000 \mathrm{~K}$.

The coefficients we have chosen in relations (6) and (7) are given in Table 1; they are representative of the behaviour of usual metals (cf. [20], Table 1). In particular, with the value chosen for $G_{0}$, the order of magnitude of the ratio $Y / G$ for metals, i.e. one percent, is satisfied;

3. constant isochoric heat capacity: $C_{v}=500 \mathrm{~J} \cdot \mathrm{kg}^{-1} . \mathrm{K}^{-1}$.

\subsection{Dominant mode and plastic strain localization lines}

We carry out tension and compression tests on plates made with the material of Section 2.2, whose initial thickness equals $2 L_{03}=2 \mathrm{~cm}$. By convention, $x_{1}$-direction is major principal stress direction $\left(\left|\Sigma_{11}\right| \geq\left|\Sigma_{22}\right|\right)$. The initial velocity gradient along $x_{1}$-axis, $D_{11}=V_{01} / L_{01}$, equals $10 \mathrm{~s}^{-1}$ in absolute value. Two tension (or compression) tests along $x_{1}$-axis differ only in the value of the velocity gradient ratio $\alpha=D_{22} / D_{11}$.

We carry out the linear stability analysis at initial time $t_{0}=0$ : then plastic strain $\varepsilon_{p}$ equals zero, and temperature is supposed to be equal to $305 \mathrm{~K}$.

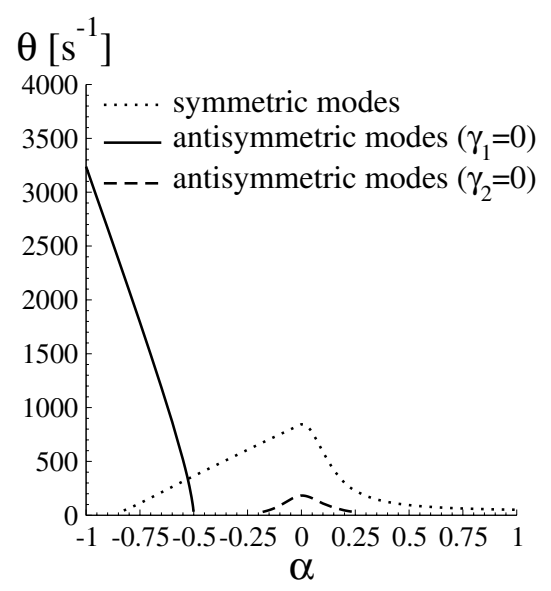

(a)

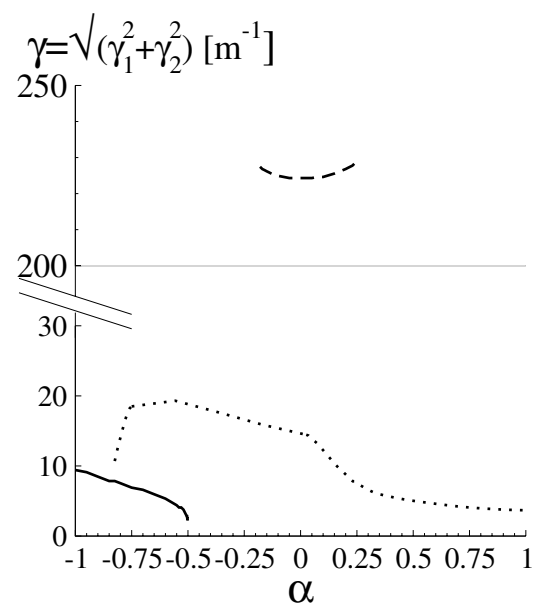

(b)

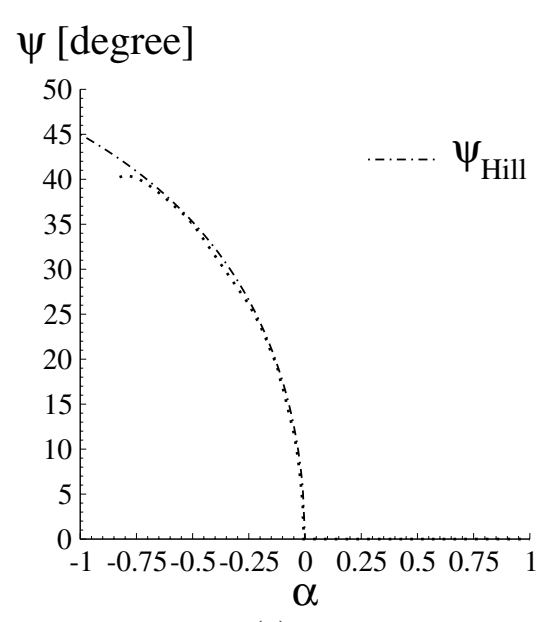

(c)

Fig. 4. Most unstable symmetric et antisymmetric modes, in tension along $x_{1}$-axis. 


$$
\text { Tension along } \mathrm{x}_{1} \text {-axis, }-1 \leq \alpha=\mathrm{D}_{22} / \mathrm{D}_{11} \leq 0
$$
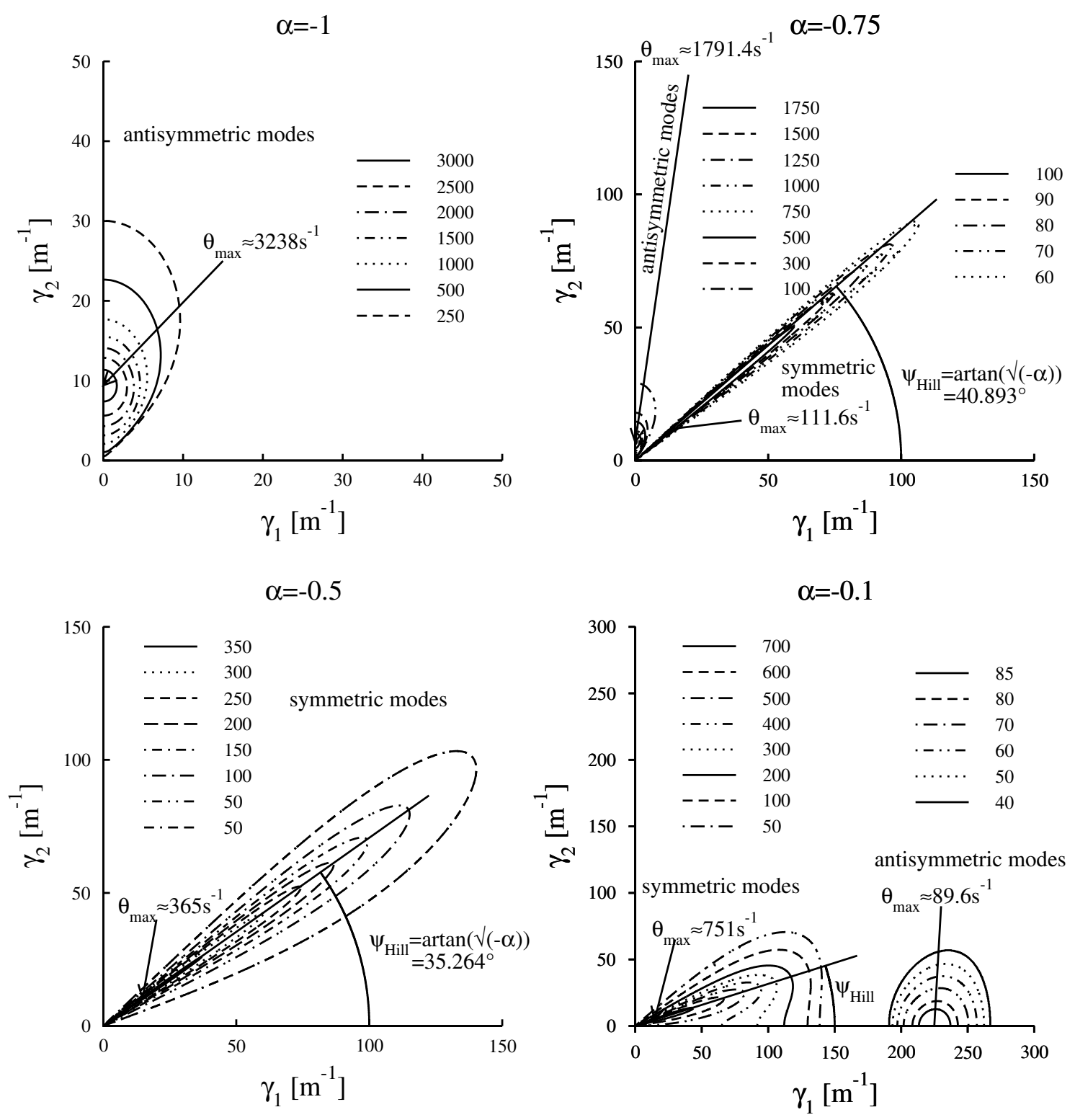

(a)

Fig. 5. Linear stability analysis of the flow of plates, loaded in tension along major principal stress direction $x_{1}$ : maps $\theta\left(\gamma_{1}, \gamma_{2}\right)$. (a) Tension along $x_{1}$-axis, compression along $x_{2}$-axis. (b) Tension simultaneously along $x_{1}$ - and $x_{2}$-axes.

\subsubsection{Tension along $x_{1}$-axis}

\section{Negative or zero velocity gradient ratio}

Loadings between uniaxial tension $(\alpha=-0.5)$ and plane tension $(\alpha=0)$.

Let us examine Figure 5a, and focus first on uniaxial tension $\left(\alpha=-0.5 ; \Sigma_{11}=Y ; \Sigma_{22}=\Sigma_{33}=0\right)$. The only unstable defects are symmetric: these are local thinnings (necks).

We draw the map $\theta\left(\gamma_{1}, \gamma_{2}\right)$, and we identify the dominant mode, i.e. the pair of wave numbers $\left(\gamma_{1}^{(d)}, \gamma_{2}^{(d)}\right)$ hav- ing the largest growth-rate. The associated wave vector $\vec{\gamma}=\gamma_{1}^{(d)} \overrightarrow{e_{1}}+\gamma_{2}^{(d)} \overrightarrow{e_{2}}$ in the plane of the loading directions is normal to the thinnest lines, where plastic strain concentrates during the linear phase of the development of necking. These are zero rate extension lines (along these lines, we have: $D_{t t}=\partial v_{t} / \partial x_{t}=0, t$ denoting the tangent direction). They are inclined at Hill's angle with respect to minor principal stress direction $x_{2}[2]$ :

$$
\psi_{\text {Hill }}=\arctan \left(\gamma_{2}^{(d)} / \gamma_{1}^{(d)}\right)=\arctan \left(\sqrt{-D_{22} / D_{11}}\right)
$$




\section{Tension along $\mathrm{x}_{1}$-axis, $0 \leq \alpha=\mathrm{D}_{22} / \mathrm{D}_{11} \leq 1$}
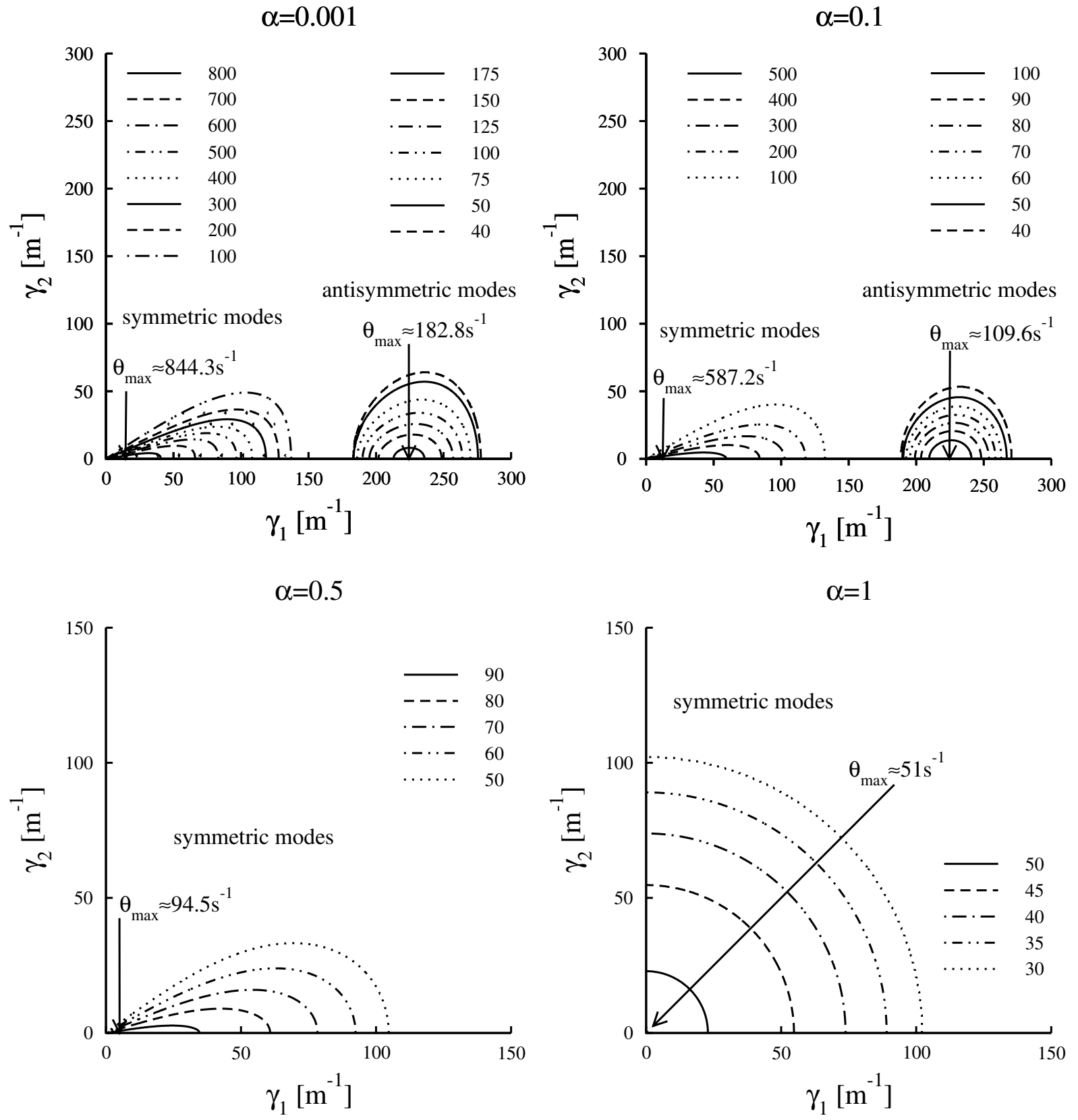

(b)

Fig. 5. Continued.

The dominant mode associated with these symmetric modes is all the less unstable that the absolute value of $\alpha$ increases (see Figs. 4a, 5a and 5b). Its orientation is given by Hill's angle (cf. Fig. 4c).

Loadings between uniaxial tension $(\alpha=-0.5)$ and simple shear $(\alpha=-1)$.

Let us examine Figure 5a once again. From uniaxial tension along $x_{1}$-axis $(\alpha=-0.5)$ to simple shear $\left(\alpha=-1 ; \Sigma_{11}=-\Sigma_{22}=Y / \sqrt{3}\right)$, we see that unstable antisymmetric modes overtake symmetric modes. The wavelength along $x_{1}$-axis of the dominant mode associated with these antisymmetric modes is infinite $\left(\gamma_{1}^{(d)}=0 \Longrightarrow\right.$ $\left.\lambda_{1}^{(d)}=\infty\right)$, and plastic strain preferably concentrates along lines parallel to $x_{1}$-axis; the wavelength $\lambda_{2}^{(d)}$ is all the shorter (and the wave number $\gamma_{2}^{(d)}$ and the 


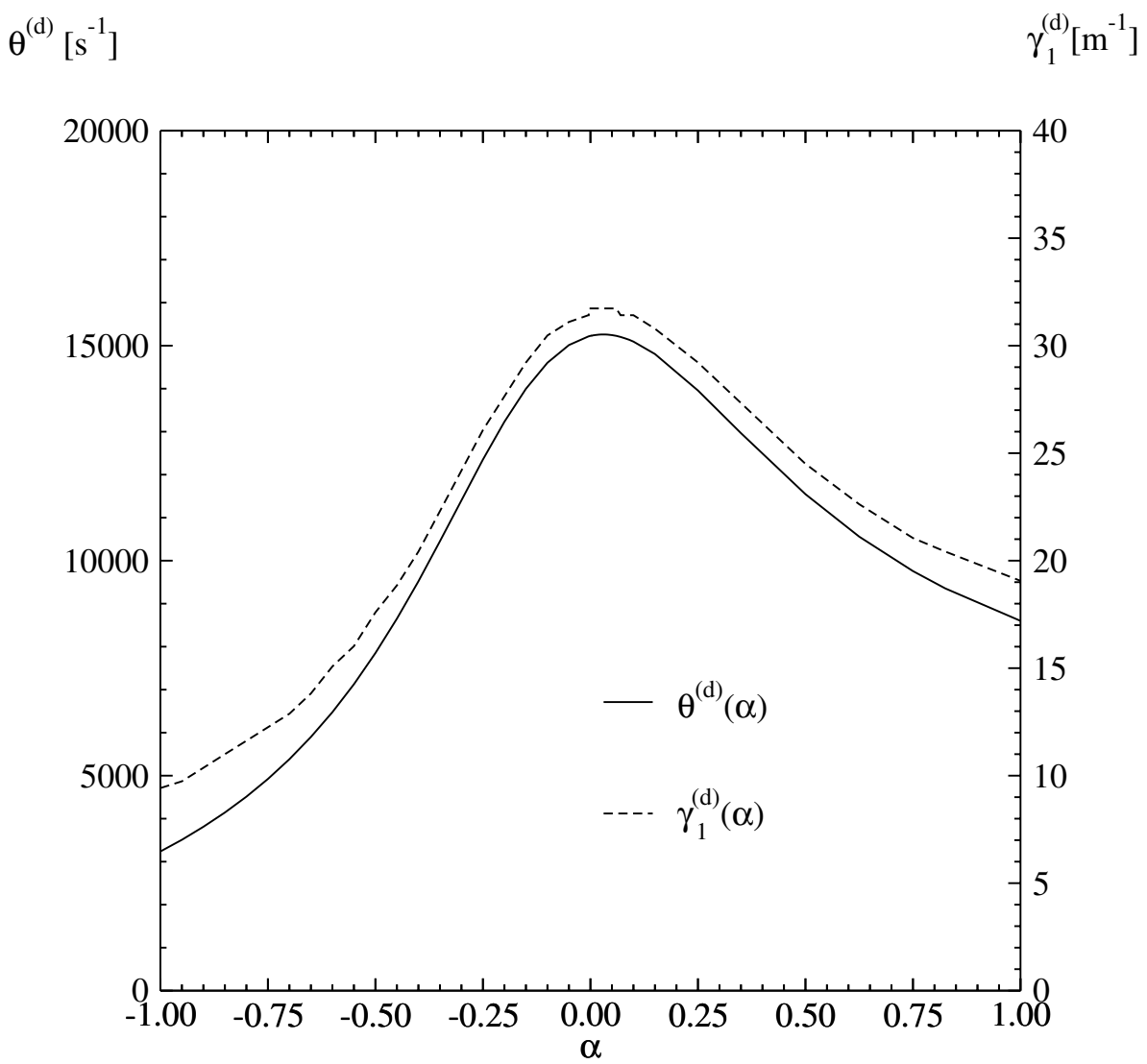

Fig. 6. Dominant mode in compression.

growth-rate $\theta^{(d)}$ all the larger) that we get nearer to simple shear (cf. Figs. 4a and 4b).

\section{Tension simultaneously along $x_{1}$ - and $x_{2}$-axes} (Fig. 5b)

The most unstable defects are symmetric with respect to the median plane of the plate: these are necks. For $\alpha<$ 1 , the wavelength along minor principal stress direction $x_{2}$ associated with the dominant mode is infinite $\left(\gamma_{2}^{(d)}=\right.$ $\left.0 \Longrightarrow \lambda_{2}^{(d)}=\infty\right)$, and plastic strain concentrates along lines parallel to $x_{2}$-axis. Plane tension $(\alpha=0)$ is the most unstable loading condition ${ }^{2}$. Getting from plane tension $(\alpha=0)$ to balanced stretching $(\alpha=1)$, the (symmetric) dominant mode becomes less and less unstable, and the associated wavelength $\lambda_{1}^{(d)}$ larger and larger (cf. Fig. 4b). For balanced stretching, the wave vector $\vec{\gamma}=\gamma_{1} \overrightarrow{e_{1}}+\gamma_{2} \overrightarrow{e_{2}}$ appears in the equations of the model only in its norm $\gamma=$ $\sqrt{\gamma_{1}^{2}+\gamma_{2}^{2}}$, and the iso- $\theta$ curves on the map $\theta\left(\gamma_{1}, \gamma_{2}\right)$ are circle quarters centred at the origin, and all orientations for localization lines are equiprobable.

Finally, in the neighbourhood of plane tension, there exist also unstable antisymmetric modes (cf. Figs. 4a, 4b, and $5 \mathrm{a}, 5 \mathrm{~b})$. Their wavelength is comparable to the thick-

\footnotetext{
${ }^{2}$ More precisely, the most unstable loading condition is in the present case such as: $\alpha \approx 0.005$.
}

ness of the plate, and is shorter than the one of the unstable symmetric modes. Due to viscous effects, they do not dominate the symmetric modes.

\subsubsection{Compression along $x_{1}$-axis (Figs. 6 and 7)}

The most unstable defects are antisymmetric with respect to the median plane of the plate. For $\alpha<1$, plastic strain concentrates preferably along lines parallel to minor principal stress direction $x_{2}$.

$\left(\gamma_{2}^{(d)}=0 \Longrightarrow \lambda_{2}^{(d)}=\infty\right)$. The flow is all the less unstable (and the localization lines all the more spaced out) that we get farther from plane strain ${ }^{3}$. For balanced compression $(\alpha=1)$, as for balanced stretching, the wave vector $\vec{\gamma}=\gamma_{1} \overrightarrow{e_{1}}+\gamma_{2} \overrightarrow{e_{2}}$ appears in the equations of the model only in its norm $\sqrt{\gamma_{1}^{2}+\gamma_{2}^{2}}$, and the iso- $\theta$ curves in the $\left(\gamma_{1}, \gamma_{2}\right)$-plane are circle quarters centred at the origin; all orientations of localization lines are equiprobable.

\footnotetext{
${ }^{3}$ In fact, as in tension, this assertion needs to be somewhat tempered; more precisely, here the most unstable loading is such as: $\alpha \approx 0.03$, but the maximum of the curve $\theta^{(d)}(\alpha)$ is very flat in the neighbourhood of plane compression.
} 


\section{Compression along $\mathrm{x}_{1}$-axis, $-1 \leq \alpha=\mathrm{D}_{22} / \mathrm{D}_{11} \leq 1$}
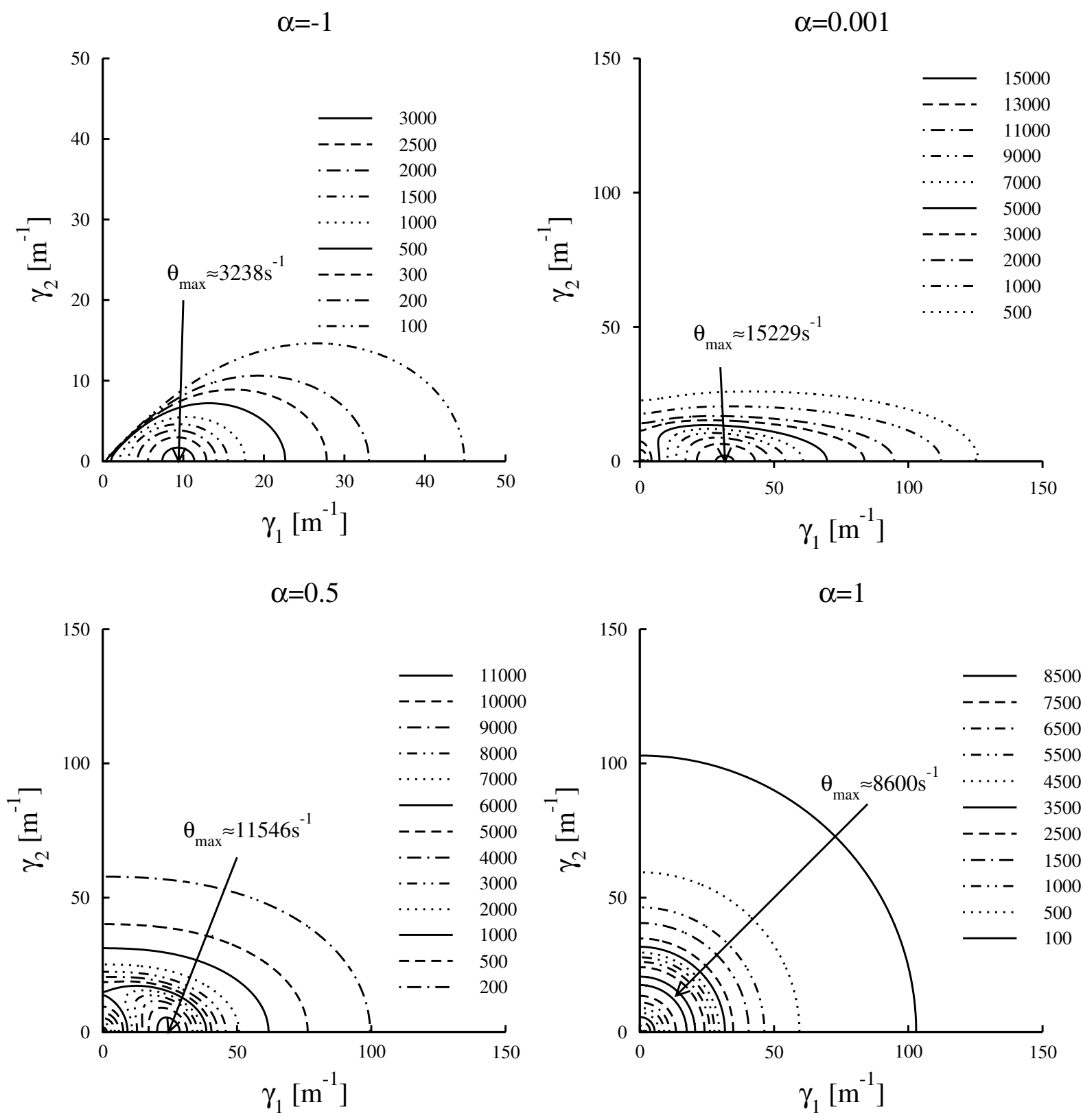

All defects are antisymmetric

Fig. 7. Linear stability analysis of the flow of plates, loaded in compression along major principal stress direction $x_{1}$ : maps $\theta\left(\gamma_{1}, \gamma_{2}\right)$.

\section{Plane tension and compression}

\subsection{Competition between symmetric and antisymmetric modes}

For plates loaded in tension (for velocity gradient ratio between -0.5 and 1 ) or in compression along major principal stress direction $x_{1}$, plane strain $\left(D_{22}=0\right.$ : the dimension of the plate along $x_{2}$-direction is infinite) is the most unstable loading condition. It has been widely investigated in the literature $[12-14,22-24]$. For us, it is a particular case of our general 3D model. Then we observe a competition between symmetric and antisymmetric modes. This competition is all the more important that viscous effects are lower. The $\theta\left(\gamma_{1}\right)$ curve is made up of a succession of branches, associated with symmetric and antisymmetric modes, alternatively. The first branch, in the field of the longest wavelengths, is associated with symmetric 

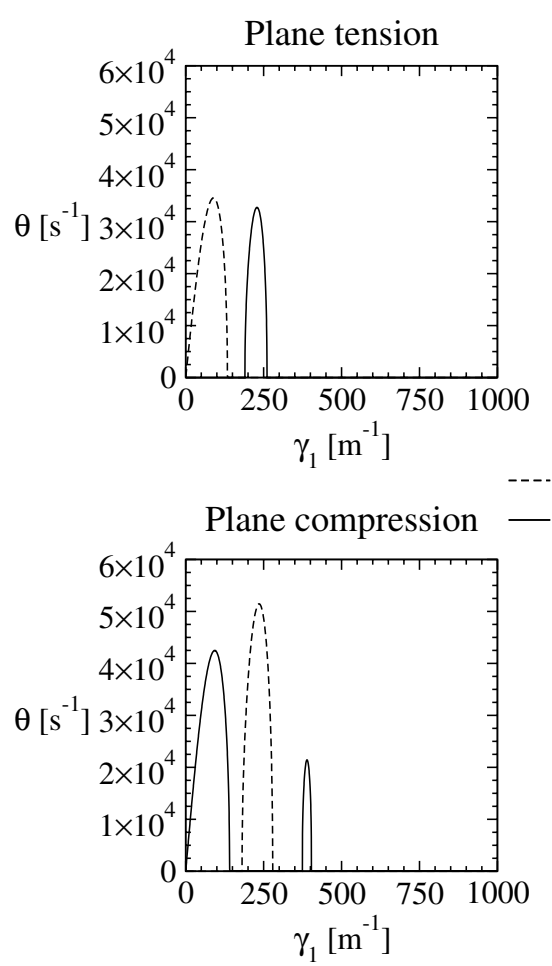

Fig. 8. Linear stability analysis of the flow of two plates loaded in plane strain, the first in tension, the second in compression. The material is not viscous, and the symmetric and antisymmetric modes are competing (stability analysis carried out at initial time $\left.t_{0}=0\right)$. Thickness: $2 L_{03}=2 \mathrm{~cm}-$ velocity gradient (in absolute value): $\left|V_{01}\right| / L_{01}=10 \mathrm{~s}^{-1}-$ mass density of the material $\rho=5000 \mathrm{~kg} \cdot \mathrm{m}^{-3}-$ yield strength $Y\left(\varepsilon_{p}\right)=$ $A+B\left(\varepsilon_{p}+\varepsilon_{i}\right)^{n}$ with: $A=B=1 G P a ; n=0.5 ; \varepsilon_{i}=1-$ shear modulus $G=100$ GPa ([16], Chap. 3).

modes in tension, and with antisymmetric modes in compression [25]. This competition has been revealed in the past, notably by Hill and Hutchinson in tension [26], and by Young in compression [27]. For very low viscous effects, the dominant mode is not always symmetric in tension, and antisymmetric in compression (cf. Fig. 8).

\subsection{First instabilities in tension}

In plane strain tension on non viscous ductile metals, the first instabilities, which are also long wavelength necking ones, arise very little time before the applied force is maximum: this result is consistent with the experimental observations of Considère [1]. Then, the growth-rate associated with the dominant mode increases, whereas the associated wavelength decreases (the number of necks increases). The instability develops clearly once the ratio $\theta / \dot{\varepsilon}_{p}$ has reached a certain threshold level (typically 10) (cf. Fig. 9) [15, 28].

\subsection{Shear bands}

In plane strain tension and compression, in the absence of viscous effects, our linear stability analysis shows
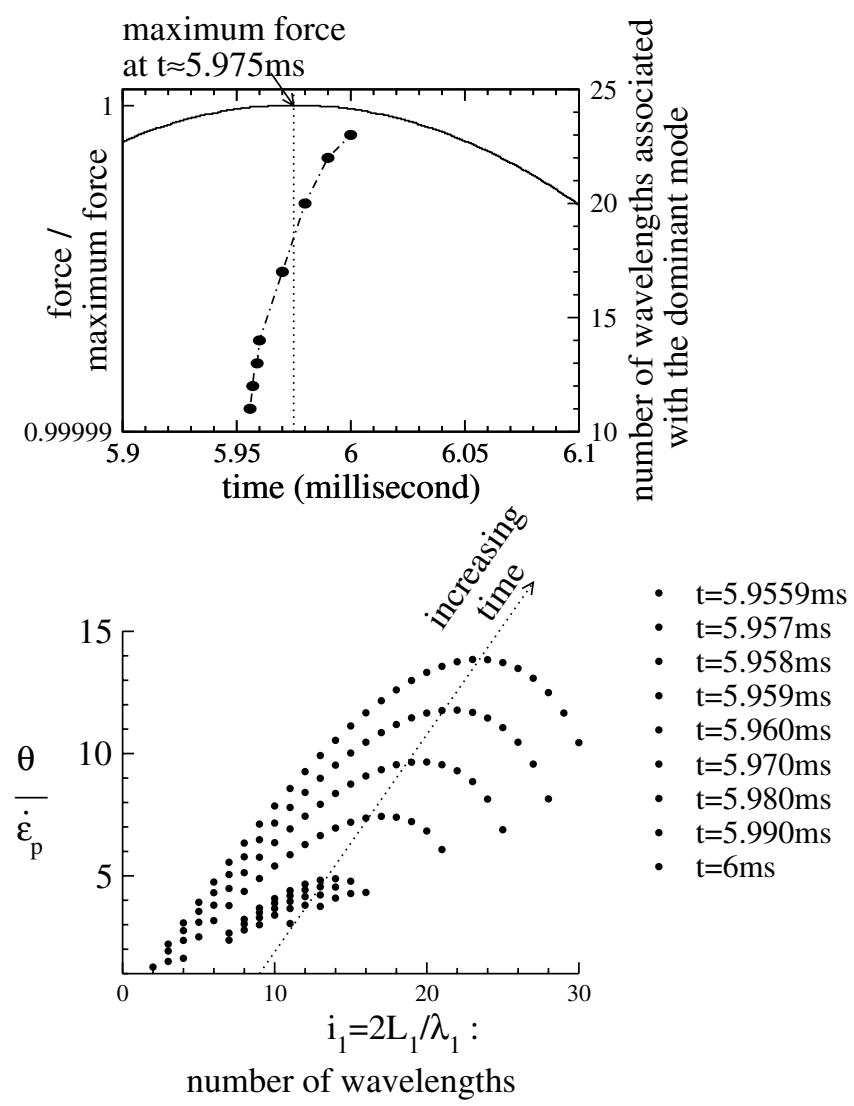

Fig. 9. Plane strain tension on a non viscous material. Initial thickness: $2 L_{03}=2 \mathrm{~cm}-$ initial velocity gradient: $V_{01} / L_{01}=$ $10 \mathrm{~s}^{-1}$ with $L_{01}=10 \mathrm{~m}$ - material: idem Figure 8, except $\varepsilon_{i}=0.1$.

that, when the effect of work-hardening on the evolution of yield strength $Y\left(\varepsilon_{p}, T\right)$ no longer sufficiently prevails over the effect of thermal softening, symmetric and antisymmetric defects having arbitrarily short wavelength develop with an infinite growth-rate $[15,16]$. Such instabilities develop as soon as the relative variation of $Y$ for a plastic strain increment $\delta \varepsilon_{p}$ satisfies the following inequality (we set: $\left.Y_{\alpha}^{\prime}=\partial Y / \partial \alpha\left(\alpha=\varepsilon_{p}, T\right)\right)$ :

$$
\begin{aligned}
\frac{\delta Y}{Y \delta \varepsilon_{p}}=\frac{Y_{\varepsilon_{p}}^{\prime}}{Y}+\frac{Y_{T}^{\prime}}{\rho C_{v}}<\frac{3}{a} \times & {\left[\frac{2}{1+\sqrt{1-\frac{a^{2}}{3}}}-1\right] } \\
& \approx \frac{a}{4} \text { setting: } a=\frac{Y}{G}
\end{aligned}
$$

Nevertheless, we have not been able to predict analytically the spatial dependence of such perturbations. We simply see that this condition for the absence of a minimal wavelength below which shorter wavelengths are all stable (cutting wavelength) coincides with the condition for the instantaneous onset of infinitely dense networks of shear bands inclined at $45^{\circ}$ with respect to the loading axis, shown by the bifurcation analysis of Hill and Hutchinson in tension [26], and the one of Young in 
$\dot{\varepsilon}_{\mathrm{p}}\left[\mathrm{s}^{-1}\right]$

25.80
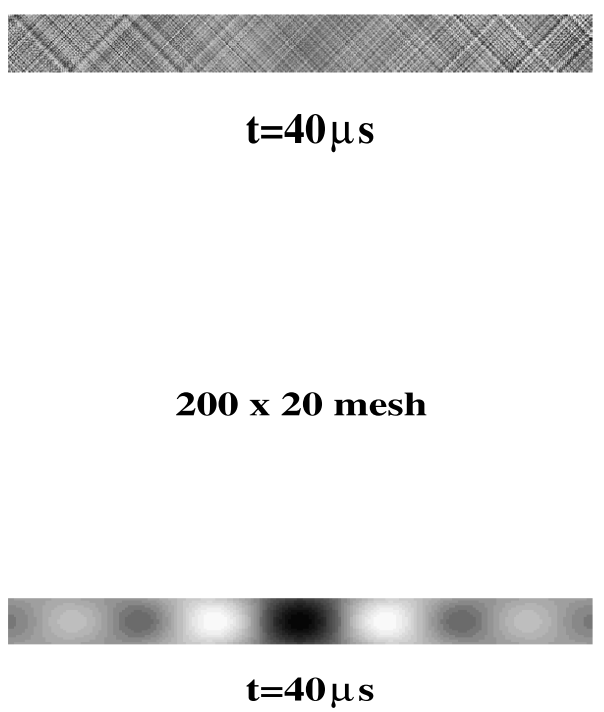

10.71

Fig. 10. Onset of a shear band network during a plane tension test on a material having constant yield strength $Y$ (then inequality (9) is well satisfied), in lieu of a long wavelength multimodal perturbation of the material velocity field introduced at initial time $t_{0}=0$ of the simulation, all the more rapidly that the mesh is refined. Initial dimensions of the plate: $2 L_{01}=20 \mathrm{~cm} ; 2 L_{03}=2 \mathrm{~cm}-$ Stretching velocity $V_{01}=1 \mathrm{~m} \cdot \mathrm{s}^{-1}-$ Mass density $\rho=5000 \mathrm{~kg} \cdot \mathrm{m}^{-3}-$ yield strength $Y=1 \mathrm{GPa}$ - shear modulus $G=100 \mathrm{GPa}$ - Two simulations of the test have been carried out, with initially square elements, with sides $100 \mu \mathrm{m}$ long for the finest mesh, and $1 \mathrm{~mm}$ long for the coarsest one [15]. We compare the plastic strain rate $\dot{\varepsilon}_{p}$ in the two simulations at time $t=40 \mu \mathrm{s}$.

compression [27] for static tests [15, 16, 29]. When the condition (9) is satisfied, we see the onset of these networks in numerical simulations, all the more rapidly and densely that the mesh is refined. Due to the absence of a physical length (and time) scale in the problem, simulations are always mesh-sensitive (see Fig. 10). The transition from an elastic perfectly plastic constitutive law $(Y=$ constant $)$ to a "sufficiently" viscous Norton's law [30] $\left(Y \propto \dot{\varepsilon}_{p}^{m}\right.$, with: $\left.m \geq 0.05\right)$ reintroduces a cutting wavelength [10-14].

\section{Conclusion and future works}

In this paper, we have shown results obtained carrying out a rigorous 3D linear stability analysis of the de- velopment of plastic instabilities during tension and compression tests on metallic plates biaxially loaded in their plane. The material is supposed to satisfy the plasticity criterion of Von Mises, and the normality flow rule.

We are undertaking to generalize our model to more complex materials, accounting for damage [31,32] and anisotropy effects [33], studying the influence of the shape of the yield surface $[5,34]$, and even texture $[35,36]$. In the limiting case of static tests on thin plates, in the absence of damage effects and for an orthotropic material obeying Hill's plasticity criterion (1948) [33], we will have to retrieve previous analytical results published in 1991 by Dudzinski and Molinari [8].

\section{References}

[1] A. Considère, Mémoire sur l'emploi du fer et de l'acier dans les constructions, Annales des Ponts et Chaussées 9 (1885) 574-775

[2] R. Hill, On discontinuous plastic states, with special reference to localized necking in thin sheets, J. Mech. Phys. Solids 1 (1952) 19-30

[3] Z. Marciniak, K. Kuczynski, Limit strains in the processes of stretch-forming sheet metal, Int. J. Mech. Sci. 9 (1967) 609-620

[4] Z. Marciniak, K. Kuczynski, T. Pokora, Influence of the plastic properties of a material on the forming limit diagram for sheet metal in tension, Int. J. Mech. Sci. 15 (1973) 789-805

[5] S. Stören, J.-R. Rice, Localized necking in thin sheets, J. Mech. Phys. Solids 23 (1975) 421-441

[6] J.-W. Hutchinson, K.-W. Neale, Sheet Necking 2: Timeindependent behavior, in: Proceedings of a Symposium on Mechanics of Sheet Metal Forming, General Motors Research Laboratories, Warren, Michigan, 17-18 october 1977, edited by D.-P. Koistinen, N.-M. Wang, Plenum Press, New York, 1978, pp. 127-150

[7] D. Dudzinski, A. Molinari, Instabilité de la déformation viscoplastique en chargement biaxial, Compte Rendu à l'Académie des Sciences de Paris 307 (1988) 1315-1321

[8] D. Dudzinski, A. Molinari, Perturbation analysis of thermoviscoplastic instabilities in biaxial loading, Int. J. Solids Struct. 27 (1991) 601-628

[9] G. Chatzigeorgiou, N. Charalambakis, Instability analysis of non-homogeneous materials under biaxial loading, Int. J. Plasticity 21 (2005) 1970-1999

[10] J.-W. Hutchinson, H. Obrecht, Tensile instabilities in strain-rate dependent materials, in Proceedings of the Fourth International Conference on Fracture ICF4, edited by D.-M.-R. Taplin, Pergamon Press, Université de Waterloo, Canada, 19-24 june 1977, Vol. 1, pp. 101116

[11] J.-W. Hutchinson, K.-W. Neale, Influence of strainrate sensitivity on necking under uniaxial tension, Acta Metallurgica 25 (1977) 839-846

[12] J.-W. Hutchinson, K.-W. Neale, A. Needleman, Sheet Necking 1: Validity of plane stress assumptions of the long-wavelength approximation, in Proceedings of a Symposium on Mechanics of Sheet Metal Forming, General Motors Research Laboratories, Warren, Michigan, 17-18 october 1977, edited by D.-P. Koistinen, N.-M. Wang, Plenum Press, New York, 1978, pp. 111-126 
[13] C. Fressengeas, A. Molinari, Instability and bifurcation in the plane tension test, Archives of Mechanics 44 (1992) 93-104

[14] C. Fressengeas, A. Molinari, Fragmentation of rapidly stretching sheets, Eur. J. Mech. A/Solids 13 (1994) 251268

[15] D. Jouve, Étude analytique de l'instabilité plastique de striction pour une plaque sollicitée en traction biaxiale, Thèse, École Polytechnique, 2010

[16] D. Jouve, Étude analytique d'instabilités plastiques pour une plaque métallique sollicitée biaxialement, en traction ou en compression : modes symétriques et antisymétriques par rapport au plan médian, Rapport CEAR 6306, 2012

[17] D. Jouve, Analytic study of the onset of plastic necking instabilities during biaxial tension tests on metallic plates, Eur. J. Mech. A/Solids 50 (2015) 59-69

[18] R. Hill, The mathematical theory of plasticity, Oxford Classic Texts in the Physical Sciences, Clarendon Press, Oxford, 1950

[19] P.-G. Hodge, The method of characteristics applied to problems of steady motion in plane plastic stress, Quart. Appl. Math. 8 (1951) 381-386

[20] G.-R. Johnson, W.-H. Cook, A constitutive model and data for metals subjected to large strains, high strain rates and high temperatures, in: Proceedings of the Seventh International Symposium on Ballistics, The Hague, The Netherlands, 19-21 april 1983, pp. 541-547

[21] D.-J. Steinberg, S.-G. Cochran, M.-W. Guinan, A constitutive model for metals applicable at high strain rate, J. Appl. Phys. 51 (1980) 1498-1504

[22] V.-B. Shenoy, L.-B. Freund, Necking bifurcations during high strain rate extension, J. Mech. Phys. Solids 47 (1999) 2209-2233

[23] S. Mercier, A. Molinari, Predictions of bifurcation and instabilities during dynamic extension, Int. J. Solids Struct. 40 (2003) 1995-2016

[24] S. Mercier, N. Granier, A. Molinari, F. Llorca, F. Buy, Multiple necking during the dynamic expansion of hemispherical metallic shells, from experiments to modelling, J. Mech. Phys. Solids 58 (2010) 955-982
[25] D. Jouve, Analytic study of the onset of plastic instabilities during plane tension and compression tests on metallic plates, Eur. J. Mech. A/Solids 47 (2014) 70-81

[26] R. Hill, J.-W. Hutchinson, Bifurcation phenomena in the plane tension test, J. Mech. Phys. Solids 23 (1975) 239 264

[27] N.-J.-B. Young, Bifurcation phenomena in the plane compression test, J. Mech. Phys. Solids 24 (1976) 77-91

[28] S. El Maï, Étude du développement des instabilités dans un anneau en expansion dynamique, Thèse, Université de Lorraine, 2014

[29] D. Jouve, Analytic study of plastic necking instabilities during plane tension tests, Eur. J. Mech. A/Solids 39 (2013) 180-196

[30] F.-H. Norton, The creep of steel at high temperatures, McGraw-Hill, 1929

[31] A.-L. Gurson, Continuum theory of ductile rupture by void nucleation and growth: Part 1 Yield criteria and flow rules for porous ductile media, Trans. ASME, J. Eng. Mater. Technol. (1977) 2-15

[32] J. Lemaitre, J.-L. Chaboche, Mécanique des matériaux solides, 2nd édition, Dunod, 1984

[33] R. Hill, A theory of the yielding and plastic flow of anisotropic metals, in: Proceedings of the Royal Society of London, Series A - Mathematical and Physical Sciences, 1948, Vol. 193, 1033, pp. 281-297

[34] J.-L. Dequiedt, Localization in elasto-plastic materials: influence of the plasticity yield surface in biaxial loading conditions, Int. J. Solids Struct. 47 (2010) 2937-2951

[35] L.-S. Tóth, D. Dudzinski, A. Molinari, Forming limit predictions with the perturbation method using stress potential functions of polycrystal viscoplasticity, Int. J. Mech. Sci. 38 (1996) 805-824

[36] N. Boudeau, J.-C. Gelin, S. Salhi, Computational prediction of the localized necking in sheet forming based on microstructural material aspects, Comput. Mater. Sci. 11 (1998) 45-64 